\title{
Image Tiling to Improve Performance of Image Retrieval Using Color Averaging Techniques
}

\author{
Dr. H.B.Kekre \\ Senior Professor \\ Computer Engineering Department, \\ MPSTME, NMIMS (Deemed-to-be \\ University), Mumbai, India
}

\author{
Sudeep D. Thepade \\ Ph.D.Research Scholar \& Associate. \\ Professor \\ Computer Engineering Department, \\ MPSTME, NMIMS (Deemed-to-be \\ University), Mumbai, India
}

\author{
Akshay Maloo \\ B.Tech (CE) Student \\ Computer Engineering Department, \\ MPSTME,NMIMS (Deemed-to-be \\ University), Mumbai, India
}

\begin{abstract}
The research in content based image retrieval has always been nurtured because of the thirst for better and faster image retrieval techniques. Reducing the feature vector size for faster image retrieval and till achieving better performance is herculean task. The paper presents 24 novel image retrieval techniques using color averaging methods on row mean, column mean, forward diagonal mean, backward diagonal mean, row \& column mean, forward \& backward diagonal mean, four tiles, sixteen tiles and 64 tiles of image. The proposed CBIR techniques are tested on generic image database having 1000 images spread across 11 categories and COIL image database having 1080 images spread across 15 categories. For each proposed CBIR technique 75 queries ( 5 per category) are fired on the generic image database and 55 queries ( 5 per category) are fired on the COIL image database. To compare the performance of image retrieval techniques average precision and recall are computed of all queries. The results have shown the performance improvement (higher precision and recall values) with proposed methods compared to all pixel data of image at reduced computations resulting in faster retrieval
\end{abstract}

\section{Keywords}

CBIR, Image Tiling, row mean, column mean, diagonal mean

\section{INTRODUCTION}

With the advancement in technology, a large amount of information in the form of images is being generated daily from a variety of sources (digital camera, digital video, scanner, the internet etc.) which have posed technical challenges to computer systems to store/transmit and index/manage image data effectively to make such collections easily accessible. Image compression deals with the challenge of storage and transmission, where significant advancements have been made $[1,4,5]$. The challenge to image indexing is studied in the context of image database $[2,6,7,10,11]$, which has become one of the promising and important research area for researchers from a wide range of disciplines like computer vision, image processing and database areas.

The thirst of better and faster image retrieval techniques is increasing day by day. Some of important applications for CBIR technology could be identified as art \& craft museums [12,14], architecture design [8,13], archaeology [3], geographic information systems [5], medical imaging [5,18], weather forecast [5,22], trademark databases [21,23], criminal investigations [24,25], image search over the Internet [9,19,20].

\subsection{Content Based Image Retrieval}

In literature the term content based image retrieval (CBIR) has been used for the first time by Kato et.al. [4], to describe his experiments into automatic retrieval of images from a database by color and shape feature. The typical CBIR system performs two major tasks $[16,17]$. The first one is feature extraction (FE), where a set of features, called feature vector, is generated to accurately represent the content of each image in the database. The second task is similarity measurement (SM), where a distance between the query image and each image in the database using their feature vectors is used to retrieve the top "closest" images [16,17,26].

For feature extraction in CBIR there are mainly two approaches [5] feature extraction in spatial domain and feature extraction in transform domain. The feature extraction in spatial domain includes the CBIR techniques based on histograms [5], BTC $[1,2,16]$, VQ $[21,25,26]$. The transform domain methods are widely used in image compression, as they give high energy compaction in transformed image [17,24]. So it is obvious to use images in transformed domain for feature extraction in CBIR [23]. But taking transform of image is time consuming. Reducing the size of feature vector using pure image pixel data in spatial domain only and till getting the improvement in performance of image retrieval is the theme of the work presented here. Many current CBIR systems use Euclidean distance [1-3,8-14] on the extracted feature set as a similarity measure. The Direct Euclidian Distance between image P and query image Q can be given as equation 1, where Vpi and Vqi are the feature vectors of image $P$ and Query image $Q$ respectively with size ' $n$ '.

$$
E D=\sqrt{\sum_{i=1}^{n}(V p i-V q i)^{2}}
$$

\section{ROW MEAN (RM) \& COLUMN MEAN (CM) $[22,27]$}

The row mean vector is the set of averages of the intensity values of the respective rows. The column mean vector is the set of averages of the intensity values of the respective columns. If fig. 1 is representing the sample image with $\mathrm{n}$ rows and $\mathrm{n}$ columns, the row and column mean vectors for this image will be as given below. 
Row Mean Vector $=$

[Avg(Row 1), Avg(Row 2), ..., Avg(Row n)]

Column Mean Vector $=$

[Avg(Col. 1), $\operatorname{Avg}($ Col. 2), ...., $\operatorname{Avg}($ Col. n)]

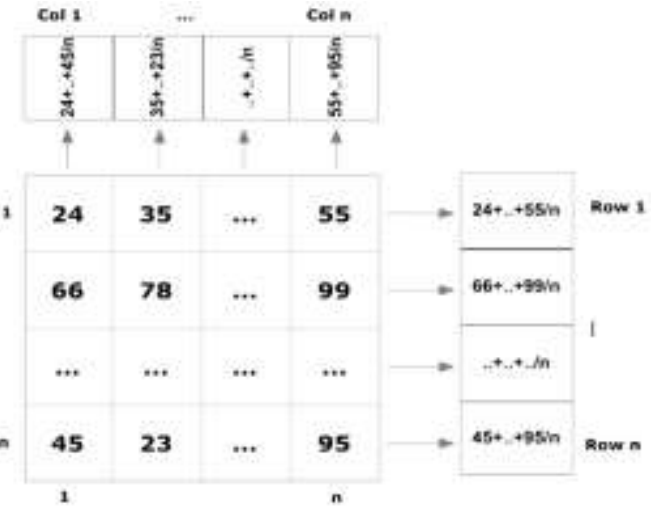

Figure 1. Row Mean and Column Mean of Sample Image

\section{FORWARD DIAGONAL MEAN (FDM) \& BACKWARD DIAGONAL MEAN (BDM)[29]}

The forward diagonal mean (FDM) vector is the set of averages of the intensity values of the diagonal elements in the direction of a forward slash. The backward diagonal mean (BDM) vector is the set of averages of the intensity values of the diagonal elements in the direction of a backward slash. If figure 2 is representing the sample image with $\mathrm{n}$ rows and $\mathrm{n}$ columns, the FDM vector is shown and figure $3 \mathrm{BDM}$ vector is shown, and the final vector for this image will be as given below.

Forward Diagonal Mean Vector =

[Avg(FDM 1), Avg(FDM 2), ..., Avg(FDM n-1)]

Backward Diagonal MeanVector $=$

[Avg(BDM. 1), Avg(BDM. 2), ..., Avg(BDM. n-1)]

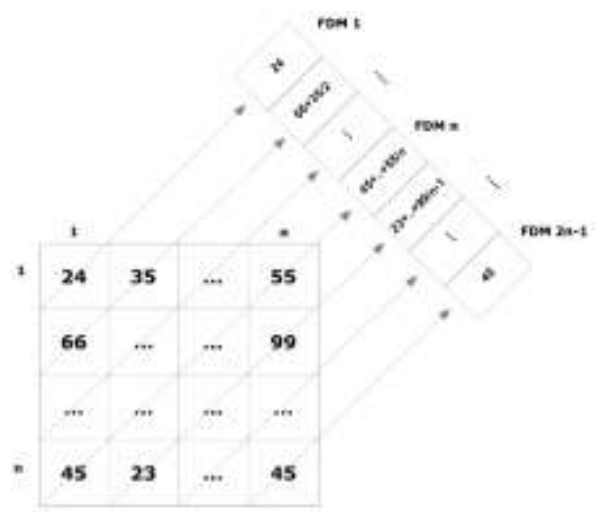

Figure 2. Forward Diagonal Mean of Sample Image

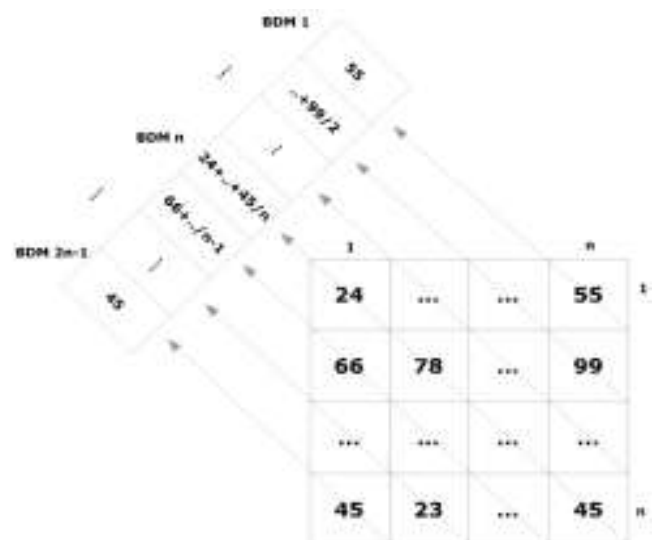

Figure 3. Backward Diagonal Mean of Sample Image

\section{IMAGE TILING}

Image Tiling [30] means dividing any given image into nonoverlapping cells or fragments. The size of each tile is such that it divides the image into $\mathrm{N}$ equal parts and also keeping the size of each tile the same. Here we have considered four, sixteen and sixty four non overlapping tiles as shown in figure 4 . The size of feature vectors for respective number of tiles is shown in table 1.

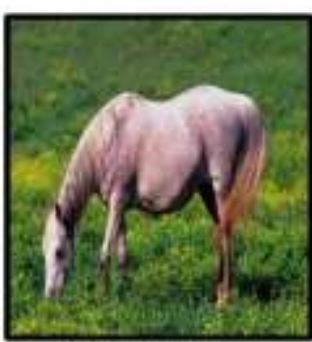

a. Single Tiles

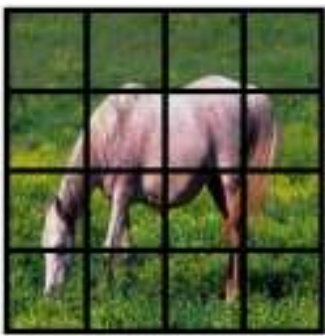

c. Sixteen Tiles

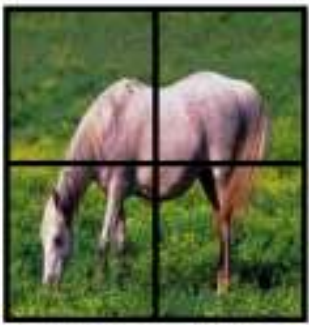

b. Four Tiles

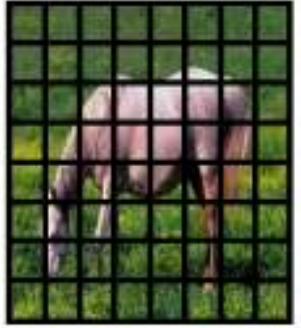

d. Sixty-Four Tiles
Figure 4. Tiling of an image into single, 4, $16 \& 64$ tiles respectively

\section{PROPOSED COLOUR AVERAGING TECHNIQUES}

The various proposed techniques are:

- All image pixels,

- Row Mean of image (RM),

- Column mean of image (CM),

- _Combination row and column mean (RCM),

- Forward diagonal mean of image (FDM),

- Backward diagonal mean of image (BDM), 
- Combination of diagonal means (FBDM).

Then the image is tiled into four, sixteen and sixty four parts and all above discussed techniques are applied for red, green and blue plane. All these techniques are used to generate feature vector by considering Colour images from the database resulting into total 24 different CBIR techniques. The advantage of using the means of images over the complete pixel data of image is reduced complexity for image retrieval with improved performance in terms of higher precision and recall values.

Table 1. Size of feature vector for NxN Image according to number of tiles

\begin{tabular}{|c|c|c|c|c|}
\hline \multirow{2}{*}{$\begin{array}{l}\text { Feature } \\
\text { Extraction } \\
\text { Techniques }\end{array}$} & \multicolumn{4}{|c|}{ No of Tiles } \\
\cline { 2 - 5 } & $\mathbf{1}$ & $\mathbf{4}$ & $\mathbf{1 6}$ & $\mathbf{6 4}$ \\
\hline Complete Image & $\mathrm{N}^{2}$ & $\mathrm{~N}^{2}$ & $\mathrm{~N}^{2}$ & $\mathrm{~N}^{2}$ \\
\hline RM/CM & $\mathrm{N}$ & $2 \mathrm{~N}$ & $4 \mathrm{~N}$ & $8 \mathrm{~N}$ \\
\hline RCM & $2 \mathrm{~N}$ & $4 \mathrm{~N}$ & $8 \mathrm{~N}$ & $16 \mathrm{~N}$ \\
\hline FDM/BDM & $2 \mathrm{~N}-1$ & $2 \mathrm{~N}-4$ & $4 \mathrm{~N}-16$ & $8 \mathrm{~N}-64$ \\
\hline FBDM & $4 \mathrm{~N}-2$ & $4 \mathrm{~N}-8$ & $8 \mathrm{~N}-32$ & $16 \mathrm{~N}-128$ \\
\hline \multicolumn{5}{|r}{} \\
\hline
\end{tabular}

\section{IMPLEMENTATION}

The implementation of the proposed CBIR techniques is done in MATLAB 7.0 using a computer with Intel Core 2 Duo Processor T8100 (2.1GHz) and 2 GB RAM. The CBIR techniques are tested on the generic image database (created using 839 images from Wang image database [15] with 161 additional images). The generic image database consists of 1000 variable size images spread across 11 categories of human being, animals, natural scenery and manmade things. The categories and distribution of the images is shown in table 3.

Table 3. Generic Image Database: Category-wise Distribution

\begin{tabular}{|c|c|c|c|}
\hline Category & Tribes & Buses & Beaches \\
\hline No.of Images & 85 & 99 & 99 \\
\hline Category & Horses & Mountains & Airplanes \\
\hline No.of Images & 99 & 61 & 100 \\
\hline Category & Dinosaurs & Elephants & Roses \\
\hline No.of Images & 99 & 99 & 99 \\
\hline Category & Monuments & Sunrise & \\
\hline No.of Images & 99 & 61 & \\
\hline
\end{tabular}

Figure 5a gives the sample generic database images from all categories of images including scenery, flowers, buses, animals, aeroplanes, monuments, tribal people. COIL image database [28] consists of total 1080 images of size $128 \times 128 \times 3$. There are 15 different categories consisting of 72 images in each categories To test the proposed method, from every class five query images are selected randomly. So in all 75 query images are used. Figure $5 b$ gives sample 15 object images of COIL image database.
To assess the retrieval effectiveness, we have used the precision and recall as statistical comparison parameters [1,2] for the proposed CBIR techniques. The standard definitions of these two measures are given by following equations.

$$
\begin{aligned}
& \operatorname{Pr} \text { ecision }=\frac{\text { Number_of_relevant_images_retrieved }}{\text { Total_number_of_images_retrieved }} \\
& \operatorname{Re} \text { call }=\frac{\text { Number_of_relevant_images_retrieved }}{\text { Total_number_of_relevent_images_in_database }}
\end{aligned}
$$

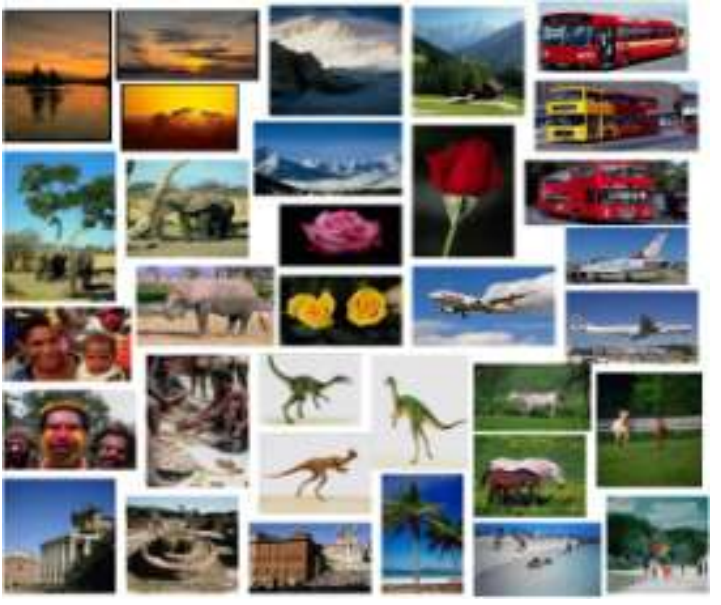

Figure 5a. Sample images of Generic Image Database [Image database contains total 1000 images with 11 categories]
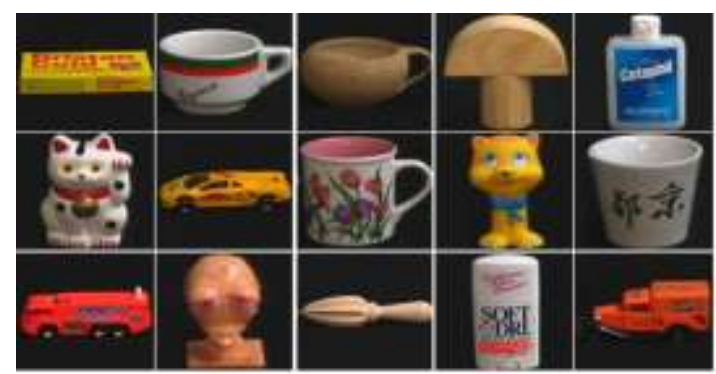

Figure 5b. Sample images of COIL Image Database [Image database contains total 1080 images with 15 categories]

Figure 5. Image Databases used for testing the performance of proposed CBIR techniques

\section{RESULTS AND DISSCUSSION}

For testing the performance of each proposed CBIR technique first five images from each category are fired on the database as queries. The average precision and average recall are computed by grouping the number of retrieved images sorted according to ascending Euclidian distances with the query image.

\subsection{Generic Database}

For testing the performance of each proposed CBIR technique, per technique 55 queries ( 5 from each category) are fired on the database of 1000 variable size generic images spread across 11 categories. The query and database image matching is done using 
Euclidian distance in RGB plane based on colour averaging technique used.

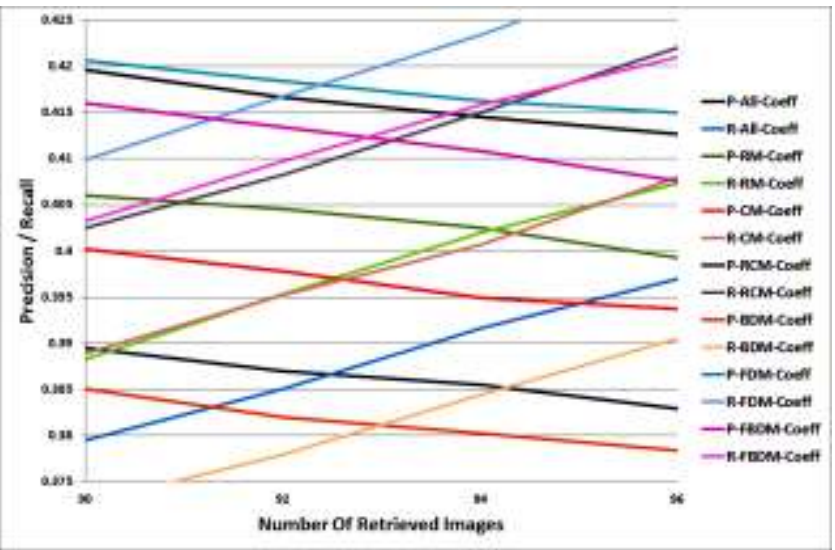

Figure 6. Crossover Point of Precision and Recall v/s Number of Retrieved Images for proposed techniques without tiling on

Generic database

Figure 6 shows the graphs of precision/recall values plotted against number of retrieved images for all proposed colour averaging based image retrieval techniques. Here forward diagonal mean (FDB) colour averaging based image retrieval technique gives the highest precision/recall crossover values specifying the best performance. Tiling has no effect when using all image pixels for image retrieval.

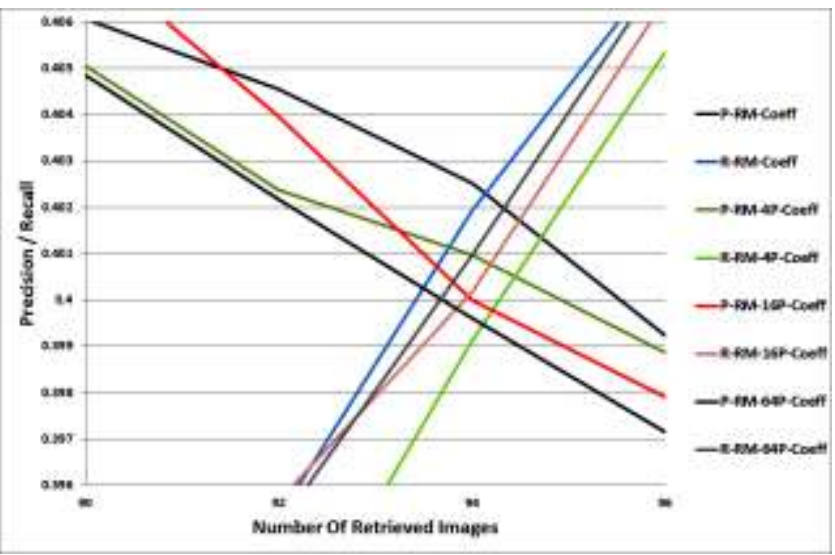

Figure 7. Crossover Point of Precision and Recall v/s Number of Retrieved Images for row mean techniques with tiling on Generic database

Figure 7 shows results obtained using tiling with row mean feature vector extraction. There is no improvement in results using tiling, the results degrade slightly as no of tiles are increased. Figure 8 shows results obtained using tiling with column mean feature vector extraction. There is significant improvement in results using tiling, the best results are obtained when the image is tiled into sixteen parts. Figure 9 shows results obtained using tiling with row \& column mean (RCM) feature vector extraction. There is no significant improvement in results when the image is tiled into 4 and 16 parts, the best results are obtained when the image is used without tiling.

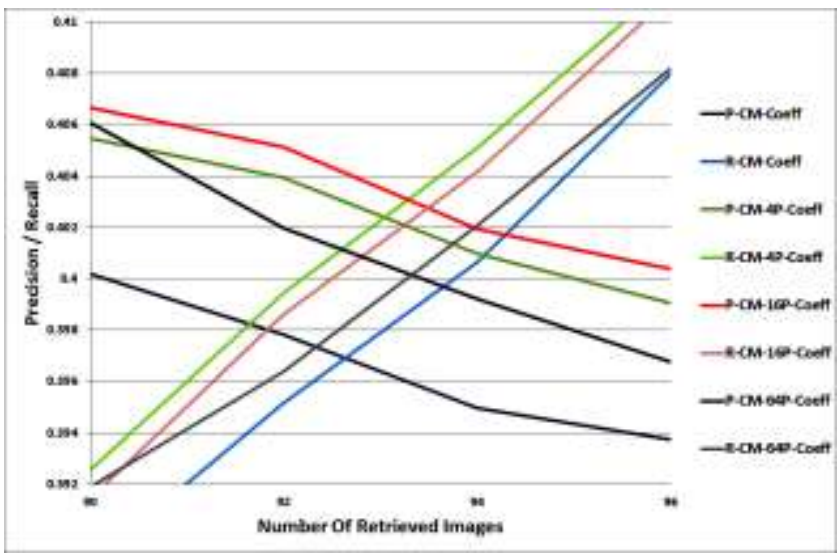

Figure 8. Crossover Point of Precision and Recall v/s Number of Retrieved Images for column mean techniques with tiling on Generic database

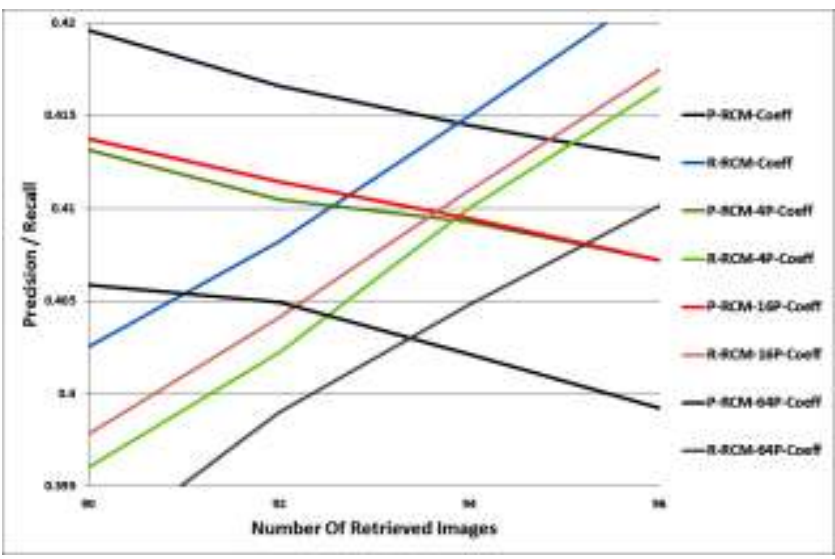

Figure 9. Crossover Point of Precision and Recall v/s Number of Retrieved Images for row \& column mean techniques with tiling on Generic database

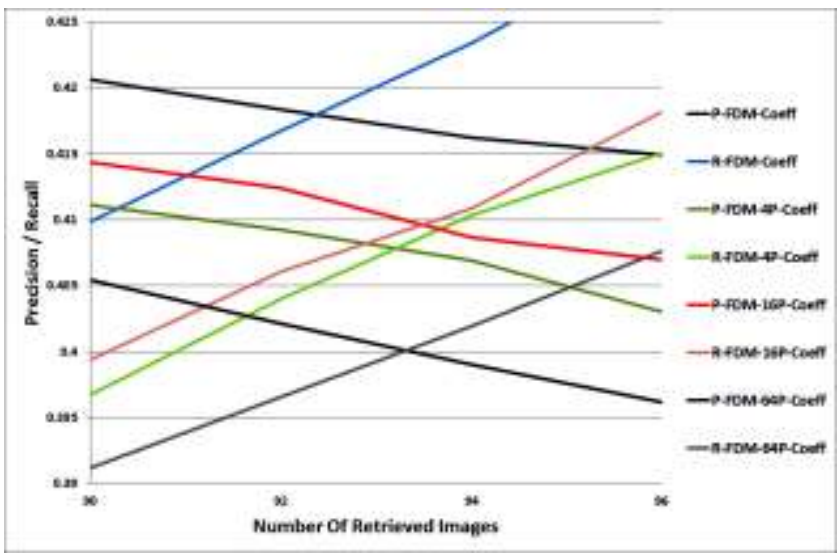

Figure 10. Crossover Point of Precision and Recall v/s Number of Retrieved Images for forward diagonal mean (FDM) techniques with tiling on Generic database 
Figure 10 shows results obtained using tiling with forward diagonal mean (FDM) feature vector extraction. There is no improvement in results using 4, 16 and 64 part tiling, the results degrade slightly as no of tiles are increased.

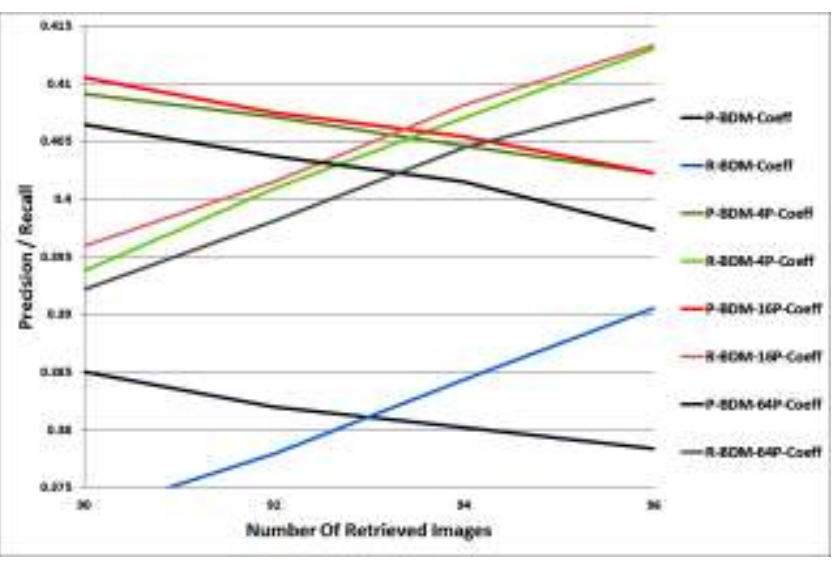

Figure 11. Crossover Point of Precision and Recall v/s Number of Retrieved Images for backward diagonal mean (BDM) techniques with tiling on Generic database

Figure 11 shows results obtained using tiling with backward diagonal mean feature vector extraction. There is significant improvement in results using 4, 16 and 64 part tiling, the best results are obtained when the image is tiled into sixteen parts. Figure 12 shows results obtained using tiling with forward \& backward diagonal mean (FBDM) feature vector extraction. There is significant improvement in results when the image is tiled to four parts.

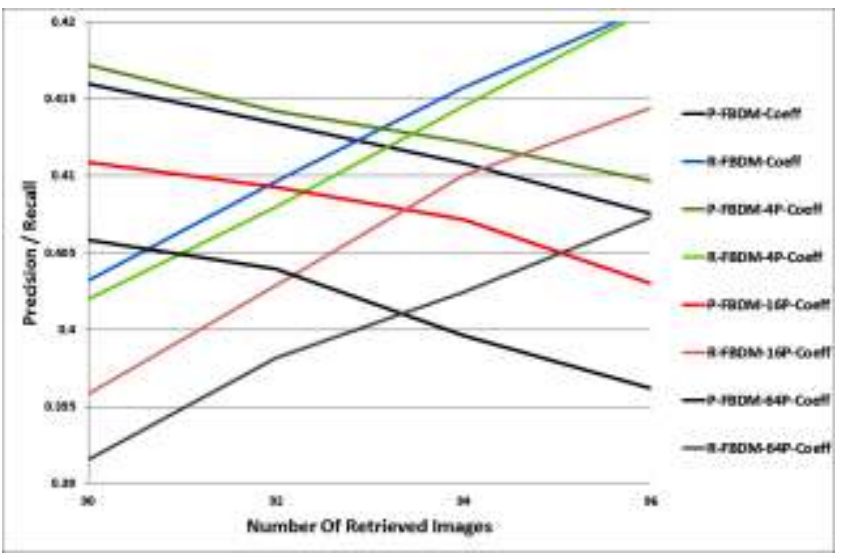

Figure 12. Crossover Point of Precision and Recall v/s Number of

Retrieved Images for forward \& backward diagonal mean (FBDM) techniques with tiling on Generic database

Figure 13 shows results obtained using tiling with all row mean and column mean feature vector extraction techniques. There is significant improvement in results using 4, 16 and 64 part tiling.

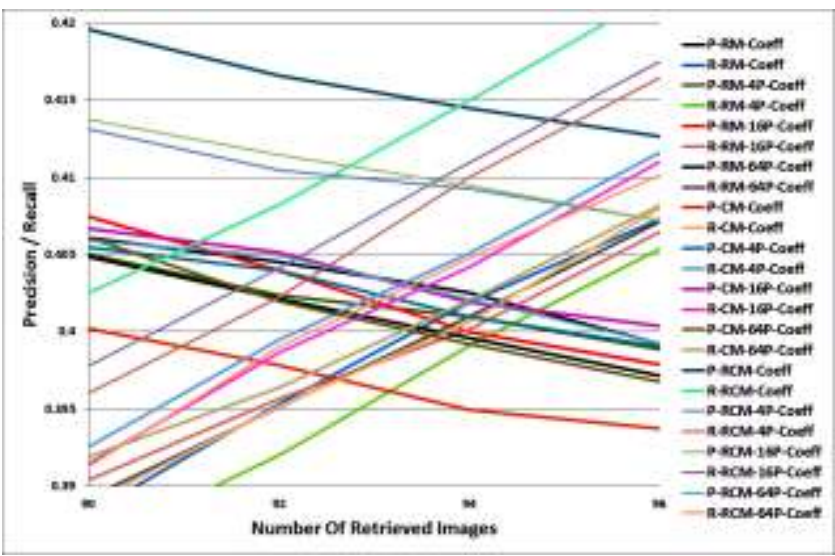

Figure 13. Crossover Point of Precision and Recall v/s Number of Retrieved Images for all row mean and column mean techniques with tiling on Generic database

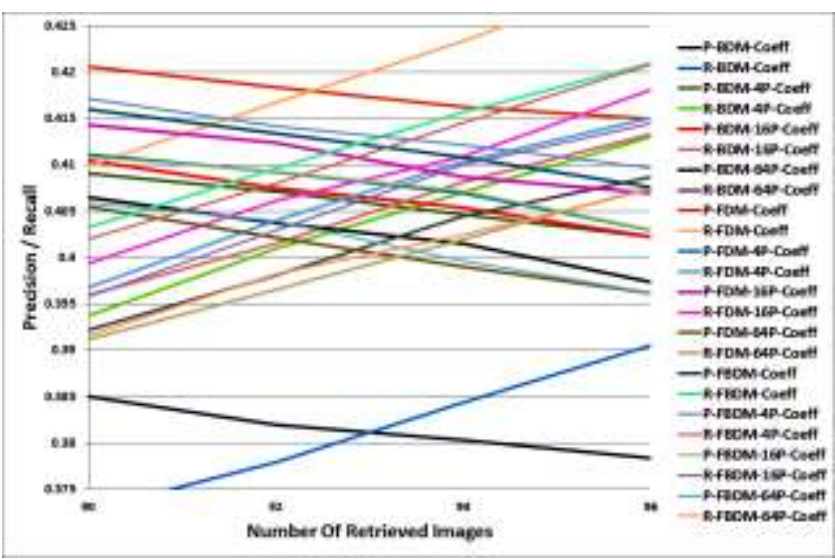

Figure 14. Crossover Point of Precision and Recall v/s Number of Retrieved Images for all forward diagonal mean and backward diagonal mean techniques with tiling

Figure 14 shows results obtained using tiling with all forward diagonal mean and backward diagonal mean feature vector extraction techniques. There is significant improvement in results using 4, 16 and 64 part tiling.

\subsection{COIL Database}

For testing the performance of each proposed CBIR technique, per technique 75 queries ( 5 from each category) are fired on the database of 1080 images spread across 15 categories. The query and database image matching is done using Euclidian distance in RGB plane based on colour averaging technique used. The average precision and average recall are computed by grouping the number of retrieved images sorted according to ascending Euclidian distances with the query image. 


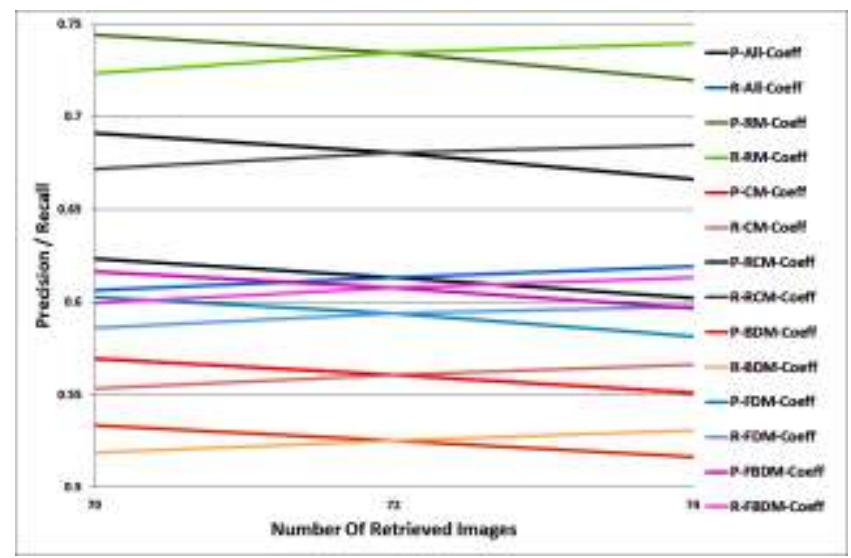

Figure 15. Crossover Point of Precision and Recall v/s Number of Retrieved Images for proposed techniques without tiling on COIL database

Figure 15 shows the graphs of precision/recall values plotted against number of retrieved images for all proposed colour averaging based image retrieval techniques. Here row mean (RM) colour averaging based image retrieval technique gives the highest precision/recall crossover values specifying the best performance. Tiling has no effect when using all image pixels for image retrieval.

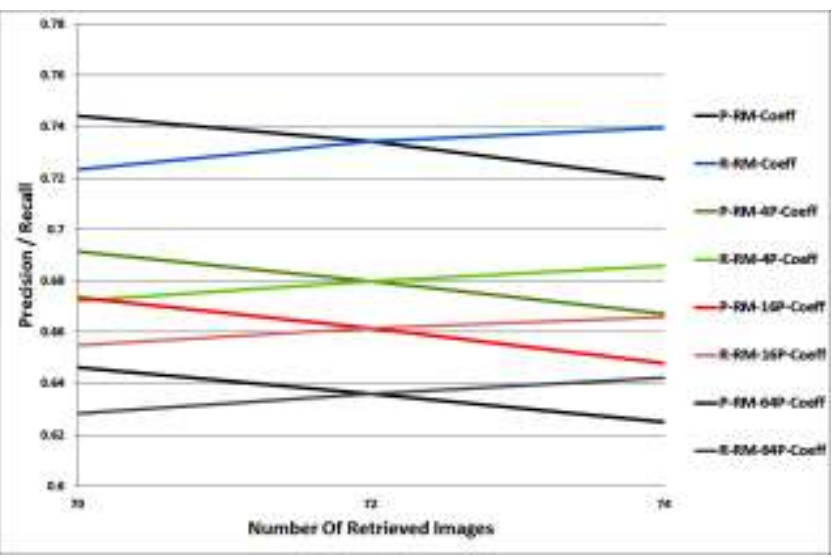

Figure 16. Crossover Point of Precision and Recall v/s Number of Retrieved Images for row mean techniques with tiling on COIL database

Figure 16 shows results obtained using tiling with row mean feature vector extraction. There is no improvement in results using tiling, the results degrade slightly as no of tiles are increased.

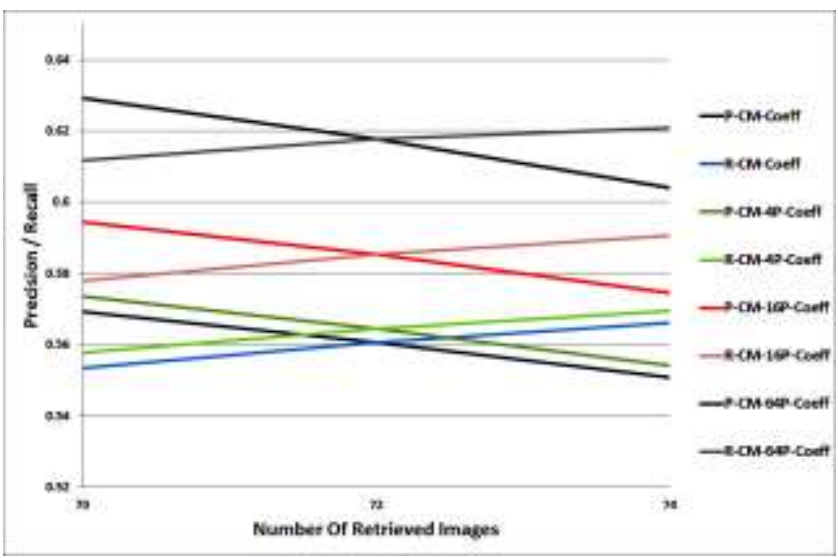

Figure 17. Crossover Point of Precision and Recall v/s Number of Retrieved Images for column mean techniques with tiling on

COIL database

Figure 17 shows results obtained using tiling with column mean feature vector extraction. There is significant improvement in results using tiling, the best results are obtained when the image is tiled into sixty four parts.

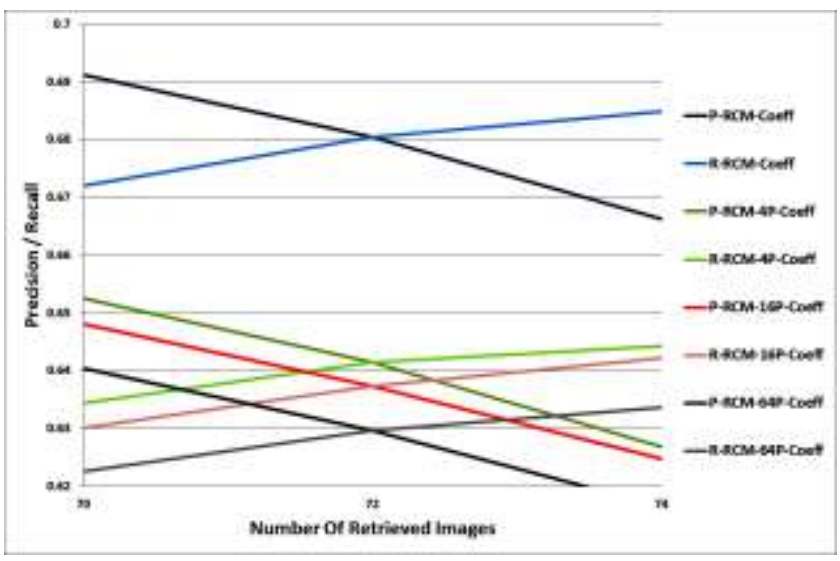

Figure 18. Crossover Point of Precision and Recall v/s Number of Retrieved Images for row \& column mean techniques with tiling on COIL database

Figure 18 shows results obtained using tiling with row \& column mean (RCM) feature vector extraction. There is no significant improvement in results when the image is tiled into 4, 16 and 64 parts, the best results are obtained when the image is used without tiling. 


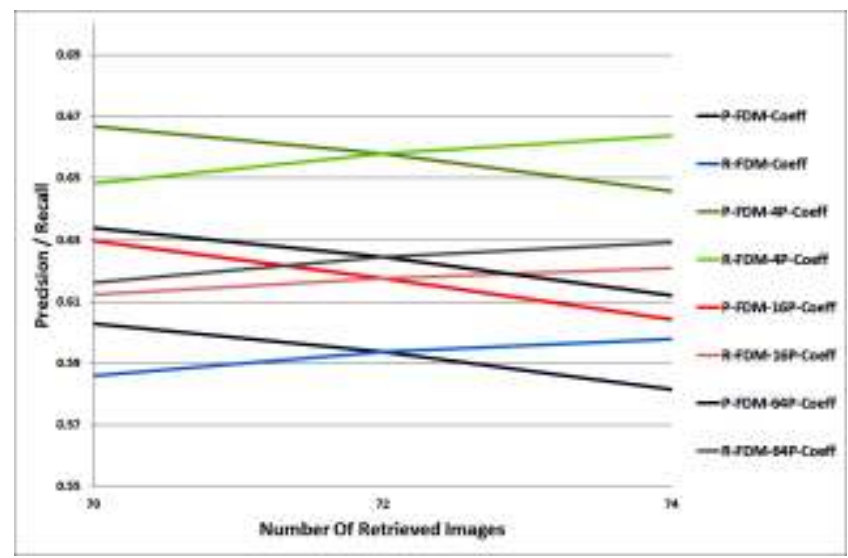

Figure 19. Crossover Point of Precision and Recall v/s Number of Retrieved Images for forward diagonal mean (FDM) techniques with tiling on COIL database

Figure 19 shows results obtained using tiling with forward diagonal mean (FDM) feature vector extraction. There is improvement in results using 4, 16 and 64 part tiling, the best results are obtained when the image is tiled into four parts. Figure 20 shows results obtained using tiling with backward diagonal mean feature vector extraction. There is significant improvement in results using 4, 16 and 64 part tiling, the best results are obtained when the image is tiled into four parts.

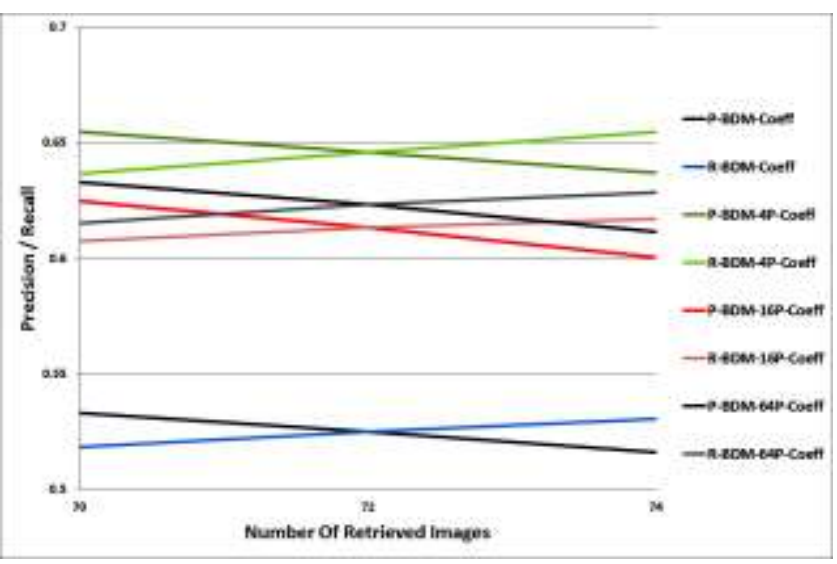

Figure 20. Crossover Point of Precision and Recall v/s Number of

Retrieved Images for backward diagonal mean (BDM) techniques with tiling on COIL database

Figure 21 shows results obtained using tiling with forward \& backward diagonal mean (FBDM) feature vector extraction. There is significant improvement in results when the image is tiled, the best using four part tiling. Figure 22 shows results obtained using tiling with all row mean and column mean feature vector extraction techniques. There is significant improvement in results using 4, 16 and 64 part tiling. Figure 23 shows results obtained using tiling with all forward diagonal mean and backward diagonal mean feature vector extraction techniques. There is significant improvement in results using 4, 16 and 64 part tiling.

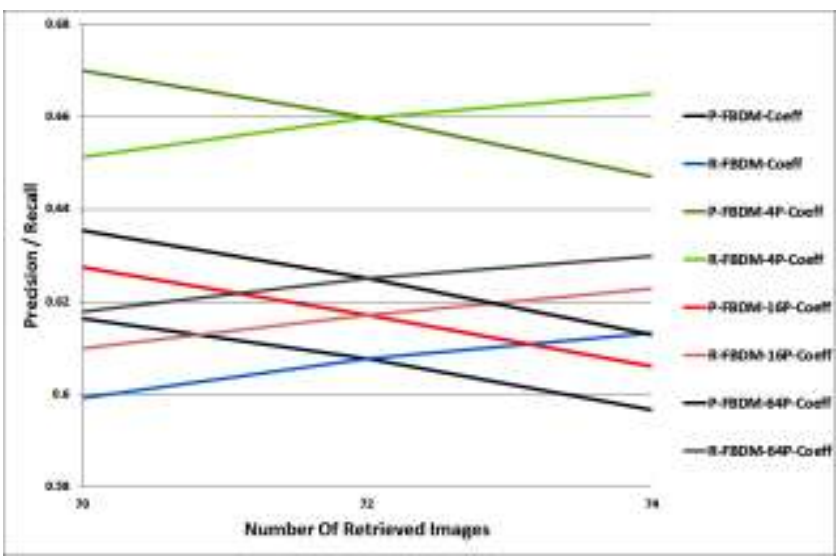

Figure 21. Crossover Point of Precision and Recall v/s Number of Retrieved Images for forward \& backward diagonal mean

(FBDM) techniques with tiling on COIL database

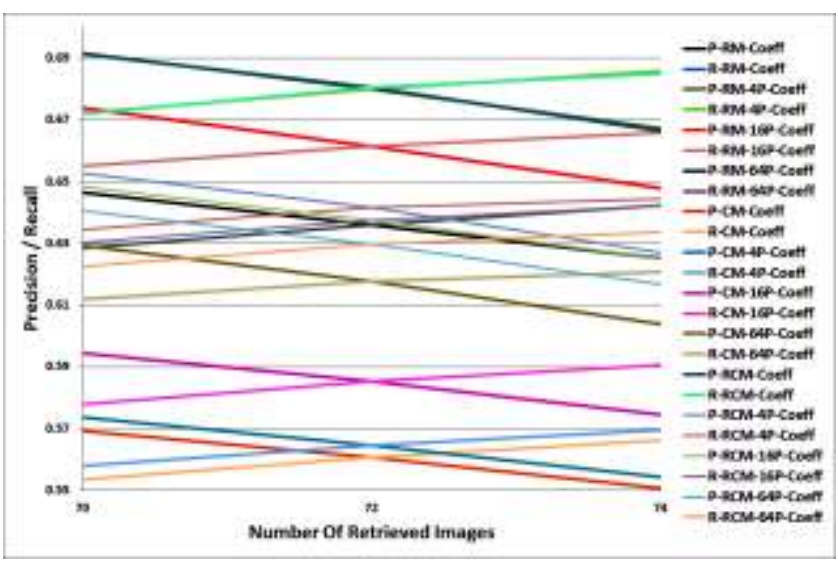

Figure 22. Crossover Point of Precision and Recall v/s Number of Retrieved Images for all row mean and column mean techniques with tiling on COIL database

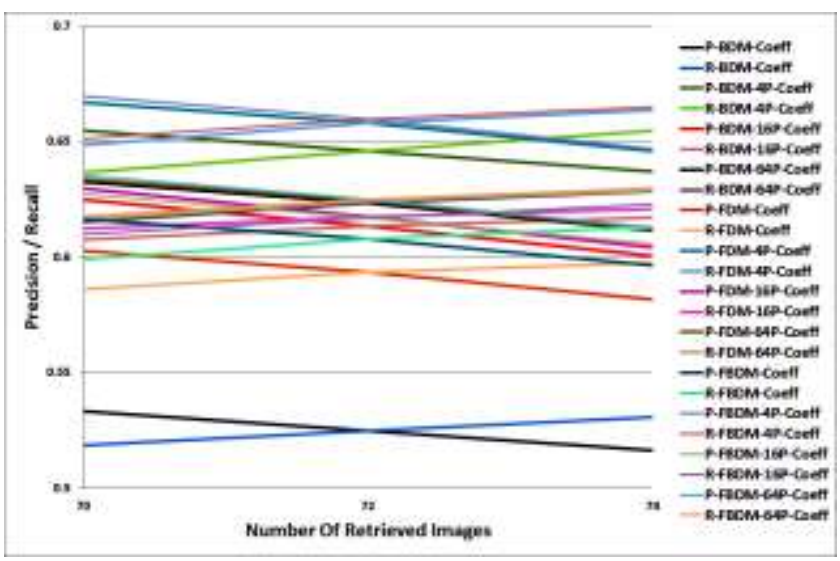

Figure 23. Crossover Point of Precision and Recall v/s Number of Retrieved Images for all forward diagonal mean and backward diagonal mean techniques with tiling 


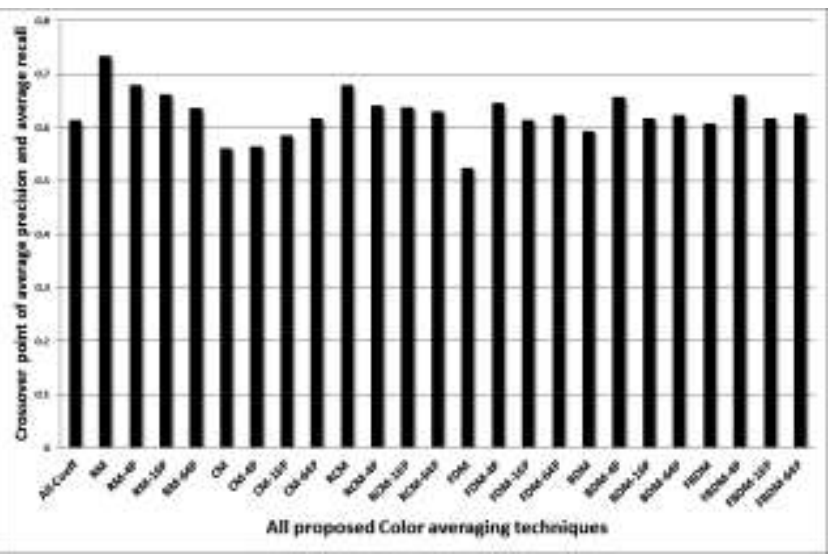

Figure 24. Performance comparison of all proposed colour averaging based image retrieval techniques on COIL image database

Figure 24 shows results for generic database row mean (RM) gives the best performance. Here it can be clearly noted that color averaging performs better than all pixel data with much reduced complexity. But image tiling is not that helpful in getting further improvements in performance.

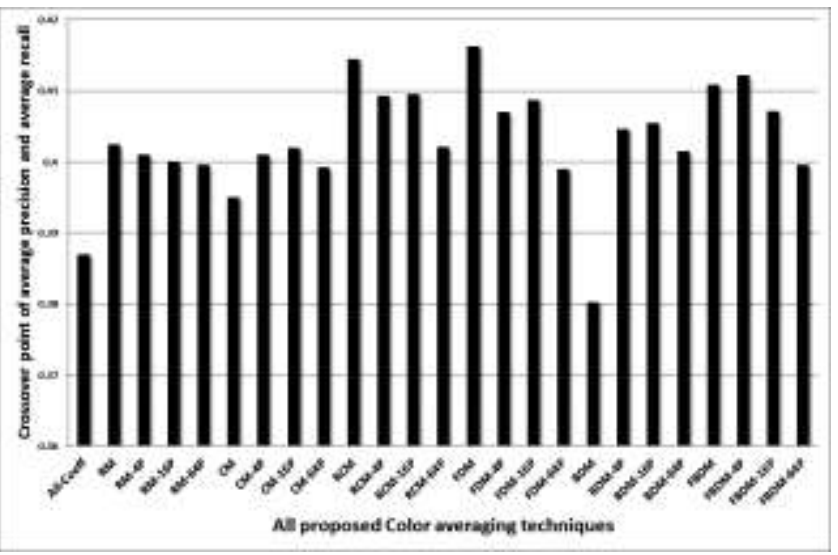

Figure 25. Performance comparison of all proposed colour averaging based image retrieval techniques on Generic image database

Figure 25 shows results for generic database forward diagonal mean (FDM) gives the best performance. Here also the trend of better performance with image averaging techniques with reduced complexity as compared to all pixel data is observed. Even in COIL image database tiling is not proving better.

\section{CONCLUSION}

The experimental results have shown that the colour averaging techniques outperform the CBIR technique using all pixel data. In generic image database forward diagonal mean gives highest precision and recall crossover value indicating best performance and all other proposed techniques perform better than all pixel data. Howsoever it is observed that the image tiling does not helps in further improvement of retrieval accuracy. The difficult task of improving the performance of content based image retrieval techniques with reduction in time complexity is achieved here with help of proposed colour averaging based CBIR techniques.

\section{REFERENCES}

[1] H.B.Kekre, Sudeep D. Thepade, "Boosting Block Truncation Coding using Kekre's LUV Color Space for Image Retrieval", WASET International Journal of Electrical, Computer and System Engineering (IJECSE), Volume 2, Number 3, pp. 172-180, Summer 2008. Available online at http://www.waset.org/ijecse/v2/v2-323.pdf

[2] H.B.Kekre, Sudeep D. Thepade, "Image Retrieval using Augmented Block Truncation Coding Techniques", ACM International Conference on Advances in Computing, Communication and Control (ICAC3-2009), pp. 384-390, 23-24 Jan 2009, Fr. Conceicao Rodrigous College of Engg., Mumbai. Is uploaded on online ACM portal.

[3] H.B.Kekre, Sudeep D. Thepade, "Scaling Invariant Fusion of Image Pieces in Panorama Making and Novel Image Blending Technique", International Journal on Imaging (IJI), www.ceser.res.in/iji.html, Volume 1, No. A08, pp. 3146, Autumn 2008.

[4] Hirata K. and Kato T. "Query by visual example - contentbased image retrieval", In Proc. of Third International Conference on Extending Database Technology, EDBT'92, 1992, pp 56-71

[5] H.B.Kekre, Sudeep D. Thepade, "Rendering Futuristic Image Retrieval System", National Conference on Enhancements in Computer, Communication and Information Technology, EC2IT-2009, 20-21 Mar 2009, K.J.Somaiya College of Engineering, Vidyavihar, Mumbai77.

[6] Minh N. Do, Martin Vetterli, "Wavelet-Based Texture Retrieval Using Generalized Gaussian Density and Kullback-Leibler Distance", IEEE Transactions On Image Processing, Volume 11, Number 2, pp.146-158, February 2002.

[7] B.G.Prasad, K.K. Biswas, and S. K. Gupta, "Region -based image retrieval using integrated color, shape, and location index", International Journal on Computer Vision and Image Understanding Special Issue: Colour for Image Indexing and Retrieval, Volume 94, Issues 1-3, April-June 2004, pp.193-233.

[8] H.B.Kekre, Sudeep D. Thepade, "Creating the Color Panoramic View using Medley of Grayscale and Color Partial Images ", WASET International Journal of Electrical, Computer and System Engineering (IJECSE), Volume 2, No. 3, Summer 2008. Available online at www.waset.org/ijecse/v2/v2-3-26.pdf.

[9] Stian Edvardsen, "Classification of Images using color, CBIR Distance Measures and Genetic Programming", Ph.D. Thesis, Master of science in Informatics, Norwegian university of science and Technology, Department of computer and Information science, June 2006. 
[10] H.B.Kekre, Tanuja Sarode, Sudeep D. Thepade, "DCT Applied to Row Mean and Column Vectors in Fingerprint Identification", In Proceedings of International Conference on Computer Networks and Security (ICCNS), 27-28 Sept. 2008, VIT, Pune.

[11] Zhibin Pan, Kotani K., Ohmi T., "Enhanced fast encoding method for vector quantization by finding an optimallyordered Walsh transform kernel", ICIP 2005, IEEE International Conference, Volume 1, pp I - 573-6, Sept. 2005.

[12] H.B.kekre, Sudeep D. Thepade, "Improving 'Color to Gray and Back' using Kekre's LUV Color Space”, IEEE International Advanced Computing Conference 2009 (IACC'09), Thapar University, Patiala, INDIA, 6-7 March 2009. Is uploaded and available online at IEEE Xplore.

[13] H.B.Kekre, Sudeep D. Thepade, "Image Blending in Vista Creation using Kekre's LUV Color Space", SPIT-IEEE Colloquium and International Conference, Sardar Patel Institute of Technology, Andheri, Mumbai, 04-05 Feb 2008.

[14] H.B.Kekre, Sudeep D. Thepade, "Color Traits Transfer to Grayscale Images", In Proc.of IEEE First International Conference on Emerging Trends in Engg. \& Technology, (ICETET-08), G.H.Raisoni COE, Nagpur, INDIA. Uploaded on online IEEE Xplore.

[15] http://wang.ist.psu.edu/docs/related/Image.orig (Last referred on 23 Sept 2008)

[16] H.B.Kekre, Sudeep D. Thepade, "Using YUV Color Space to Hoist the Performance of Block Truncation Coding for Image Retrieval", IEEE International Advanced Computing Conference 2009 (IACC'09), Thapar University, Patiala, INDIA, 6-7 March 2009.

[17] H.B.Kekre, Sudeep D. Thepade, Archana Athawale, Anant Shah, Prathmesh Verlekar, Suraj Shirke,"Energy Compaction and Image Splitting for Image Retrieval using Kekre Transform over Row and Column Feature Vectors", International Journal of Computer Science and Network Security (IJCSNS),Volume:10, Number 1, January 2010, (ISSN: 1738-7906) Available at www.IJCSNS.org.

[18] H.B.Kekre, Sudeep D. Thepade, Archana Athawale, Anant Shah, Prathmesh Verlekar, Suraj Shirke, "Walsh Transform over Row Mean and Column Mean using Image Tiling and Energy Compaction for Image Retrieval", International Journal on Computer Science and Engineering (IJCSE), Volume 2S, Issue1, January 2010, (ISSN: 09753397). Available online at www.enggjournals.com/ijcse.

[19] H.B.Kekre, Sudeep D. Thepade, "Image Retrieval using Color-Texture Features Extracted from Walshlet Pyramid", ICGST International Journal on Graphics, Vision and Image Processing (GVIP), Volume 10, Issue I, Feb.2010, pp.9-18, Available online www.icgst.com/gvip/Volume10/Issue1/P1150938876.html

[20] H.B.Kekre, Sudeep D. Thepade, "Color Based Image Retrieval using Amendment Block Truncation Coding with YCbCr Color Space", International Journal on Imaging (IJI), Volume 2, Number A09, Autumn 2009, pp. 2-14. Available online at www.ceser.res.in/iji.html (ISSN: 0974-0627).
[21] H.B.Kekre, Tanuja Sarode, Sudeep D. Thepade, "ColorTexture Feature based Image Retrieval using DCT applied on Kekre's Median Codebook", International Journal on Imaging (IJI), Volume 2, Number A09, Autumn 2009,pp. 55-65. Available online at www.ceser.res.in/iji.html (ISSN: 0974-0627).

[22] H.B.Kekre, Sudeep D. Thepade, Akshay Maloo "Performance Comparison for Face Recognition using PCA, DCT \&WalshTransform of Row Mean and Column Mean", ICGST International Journal on Graphics, Vision and Image Processing (GVIP), Volume 10, Issue II, Jun.2010, pp.9-18, Available online at http://209.61.248.177/gvip/Volume10/ Issue2/P1181012028.pdf..

[23] H.B.Kekre, Sudeep D. Thepade, "Improving the Performance of Image Retrieval using Partial Coefficients of Transformed Image", International Journal of Information Retrieval, Serials Publications, Volume 2, Issue 1, 2009, pp. 72-79 (ISSN: 0974-6285)

[24] H.B.Kekre, Sudeep D. Thepade, Archana Athawale, Anant Shah, Prathmesh Verlekar, Suraj Shirke, "Performance Evaluation of Image Retrieval using Energy Compaction and Image Tiling over DCT Row Mean and DCT Column Mean", Springer-International Conference on Contours of Computing Technology (Thinkquest-2010), Babasaheb Gawde Institute of Technology, Mumbai, 13-14 March 2010, The paper will be uploaded on online Springerlink.

[25] H.B.Kekre, Tanuja K. Sarode, Sudeep D. Thepade, Vaishali Suryavanshi,"Improved Texture Feature Based Image Retrieval using Kekre's Fast Codebook Generation Algorithm", Springer-International Conference on Contours of Computing Technology (Thinkquest-2010), Babasaheb Gawde Institute of Technology, Mumbai, 13-14 March 2010, The paper will be uploaded on online Springerlink.

[26] H.B.Kekre, Tanuja K. Sarode, Sudeep D. Thepade, "Image Retrieval by Kekre's Transform Applied on Each Row of Walsh Transformed VQ Codebook", (Invited), ACMInternational Conference and Workshop on Emerging Trends in Technology (ICWET 2010), Thakur College of Engg. And Tech., Mumbai, 26-27 Feb 2010, The paper is invited at ICWET 2010. Also will be uploaded on online ACM Portal.

[27] H.B.Kekre, Tanuja Sarode, Sudeep D. Thepade, "DCT Applied to Row Mean and Column Vectors in Fingerprint Identification", In Proceedings of Int. Conf. on Computer Networks and Security (ICCNS), 27-28 Sept. 2008, VIT, Pune.

[28] S. Nene, S.Nayar, \& H. Murase. Columbia object image library (COIL-100). Technical report, CUCS-006-96, Feb 1996 http://www1.cs.columbia.edu/CAVE/software/softlib/ coil-100.php

[29] H.B.Kekre, Sudeep D. Thepade, AkshayMaloo, "Query by Image Content Using Colour Averaging Techniques", for International Journal of Engineering Science and Technology (IJEST), Volume 2, Issue 6, 2010. Available online at http://www.ijest.info 
[30] H.B.Kekre, Sudeep D. Thepade, Archana Athawale, Anant Shaha, Prathmesh Verlekar, Suraj Shirke, "Image Retrieval using DCT on Row Mean, Column Mean and Both with Image Tiling", ACM-International Conference and Workshop on Emerging Trends in Technology (ICWET 2010), TCET, Mumbai, 26-27 Feb 2010, The paper will be uploaded on online ACM Portal.

\section{Author Biographies}

Dr. H. B. Kekre has received B.E. (Hons.) in Telecomm. Engineering. from Jabalpur University in 1958, M.Tech (Industrial Electronics) from IIT Bombay in 1960, M.S.Engg. (Electrical Engg.) from University of Ottawa in 1965 and Ph.D. (System Identification) from IIT Bombay in $1970 \mathrm{He}$ has worked as Faculty of Electrical Engg. and then HOD Computer Science and Engg. at IIT Bombay. For 13 years he was working as a professor and head in the Department of Computer Engg. At Thadomal Shahani Engineering. College, Mumbai. Now he is Senior Professor at MPSTME, SVKM's NMIMS University. He has guided $17 \mathrm{Ph} . D s$, more than 100 M.E./M.Tech and several B.E./B.Tech projects. His areas of interest are Digital Signal processing, Image Processing and Computer Networking. He has more than 300 papers in National / International Conferences and Journals to his credit. He was Senior Member of IEEE. Presently $\mathrm{He}$ is Fellow of IETE and Life Member of ISTE Recently nine students working under his guidance have received best paper awards. Currently 10 research scholars are pursuing $\mathrm{Ph} . \mathrm{D}$. program under his guidance.
Sudeep D. Thepade has Received B.E.(Computer) degree from North Maharashtra University with Distinction in 2003. M.E. in Computer Engineering from University of Mumbai in 2008 with Distinction, currently pursuing Ph.D. from SVKM's NMIMS, Mumbai. He has about than 07 years of experience in teaching and industry. He was Lecturer in Dept. of Information Technology at Thadomal Shahani Engineering College, Bandra (w), Mumbai for nearly 04 years. Currently working as Associate Professor in Computer Engineering at Mukesh Patel School of Technology Management and Engineering, SVKM's NMIMS University, Vile Parle(w), Mumbai, INDIA. He is member of International Association of Engineers (IAENG) and International Association of Computer Science and Information Technology (IACSIT), Singapore. His areas of interest are Image Processing and Computer Networks. He has about 75 papers in National/International Conferences/Journals to his credit with a Best Paper Award at International Conference SSPCCIN-2008, Second Best Paper Award at ThinkQuest-2009 National Level paper presentation competition for faculty and Best Paper Award at Springer International Conference ICCCT-2010.

Akshay Maloo is currently pursuing B.Tech. (CS) from MPSTME, NMIMS University, Mumbai. His areas of interest are Artificial intelligence, Image Processing, Computer Networks and Security. $\mathrm{He}$ has 9 papers in National/International Conferences/Journals to his credit. 